\title{
Medical Diagnostic Ultrasound
}

\author{
E. Carr Everbach \\ Swarthmore College, Swarthmore, PA, USA
}

\begin{abstract}
Although the roots of medical ultrasound lie in military technology, many of the past decade's advances have come from exploiting new understandings in the physics of mammalian tissues.
\end{abstract}

NOTE: The above article originally appeared in the March 2007 edition of Physics Today. It has been reprinted here with the permission of the American Institute of Physics. Copyright 2007, American Institute of Physics

As early as $250 \mathrm{BCE}$, captains of ancient Greek ships would drop lead weights overboard to provide an estimate of water depth. They would count until those "sounders" produced an audible thud and in that way measure the propagation time of the falling weight. Even though the practice has given way to other technologies for sounding, one still hears the phrase "to sound something out." In the 17 th century, Isaac Newton became fascinated with sound propagation and was one of the first to describe relationships between the speed of sound and measurable properties of the propagation medium, such as density and pressure. Section 8 of Book 2 of the Principia, for example, is devoted to "the motion propagated through fluids" and includes the proposition that the sound speed is given by the square root of the ratios of the "elastic force" to the density of the medium.

Newton's calculations, however, contained a systematic error for which he could not account, so his estimates of sound speed were always lower than measured values. It took until the advent of thermodynamics in the 19th century for Pierre Simon Laplace and other scientists to discover that acoustic propagation is usually adiabatic rather than isothermal. That is because free propagation does not allow sufficient time for heat exchange between compressive and rarefactive portions of the sound wave. Only in the boundary layer adjacent to a solid surface is the process approximately isothermal, a fact exploited in creating refrigerators whose only moving part is a loudspeaker (see PHYSICS TODAY, June 1999, page 18).

\section{THE DAWN OF ULTRASOUND}

Soon after the sinking of the Titanic, piezoelectric materials such as quartz or Rochelle salt were fashioned into acoustic sources whose thickness could be altered slightly by an imposed voltage. Thus underwater "sound navigation and ranging," or sonar, became possible. During World War II, both sonar and its cousin radar (radio detecting and ranging) grew in military importance; after the war they became important to civilian commerce. Sonar's familiar ping enabled vessels to use time-of-flight measurements of echoes to estimate target range and size. Modern biomedical ultrasound began during and immediately after World War II as an extension of sonar to higher frequencies and to the human body.

In the 1950 s a single piezoelectric crystal was first employed in contact with skin to launch an acoustic wave into human tissue. The wave consisted of several cycles at a frequency of $20 \mathrm{kHz}$, beyond the capacity of human hearing. A series of reflections returned to the crystal at different times because of mismatches of acoustic impedance (density $\mathrm{x}$ sound speed) between organs or between organs and bone.

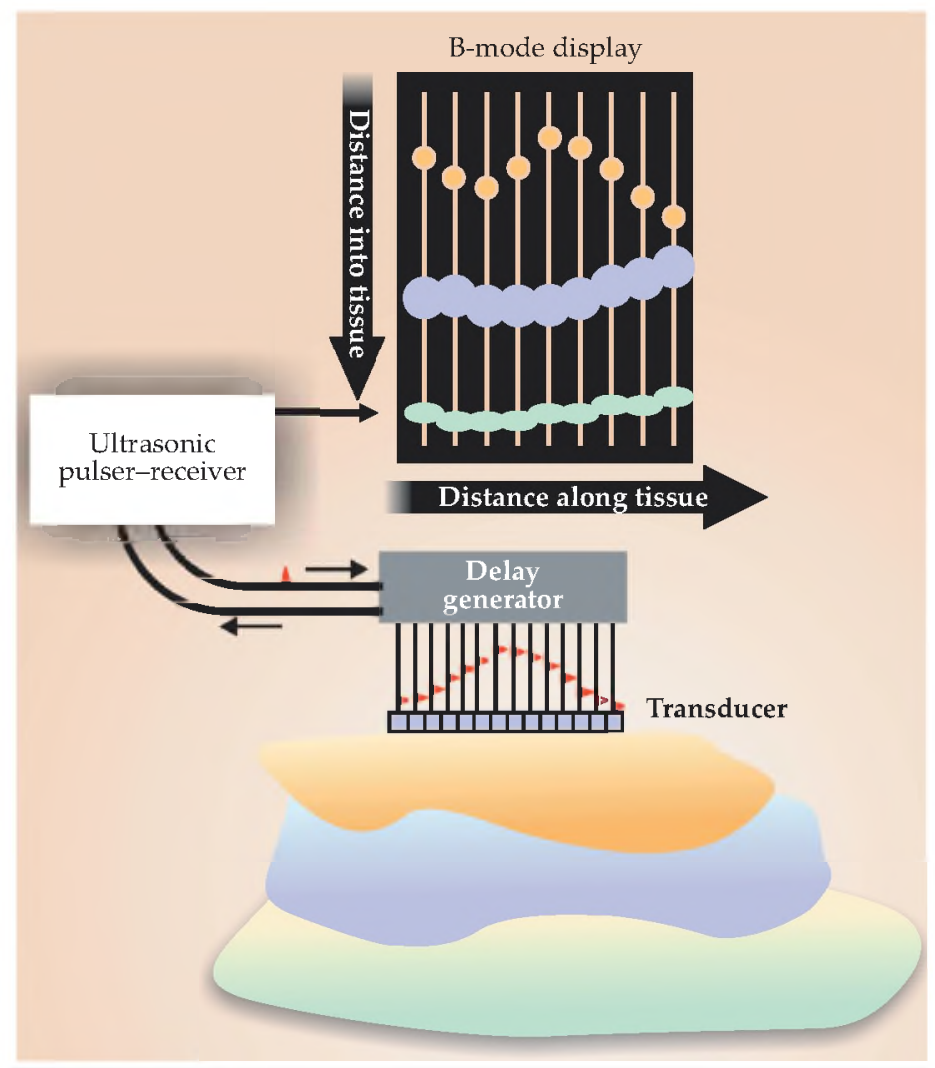

Figure 1. In a B-mode ultrasound image, a transducer scans along a tissue surface and the amplitudes of the reflected ultrasound pulses are encoded as brightness. The display plane is defined by the ultrasound beam and the scanning direction. (Courtesy of Pierre D. Mourad and Shahram Vaezy, University of Washington, Seattle.) 


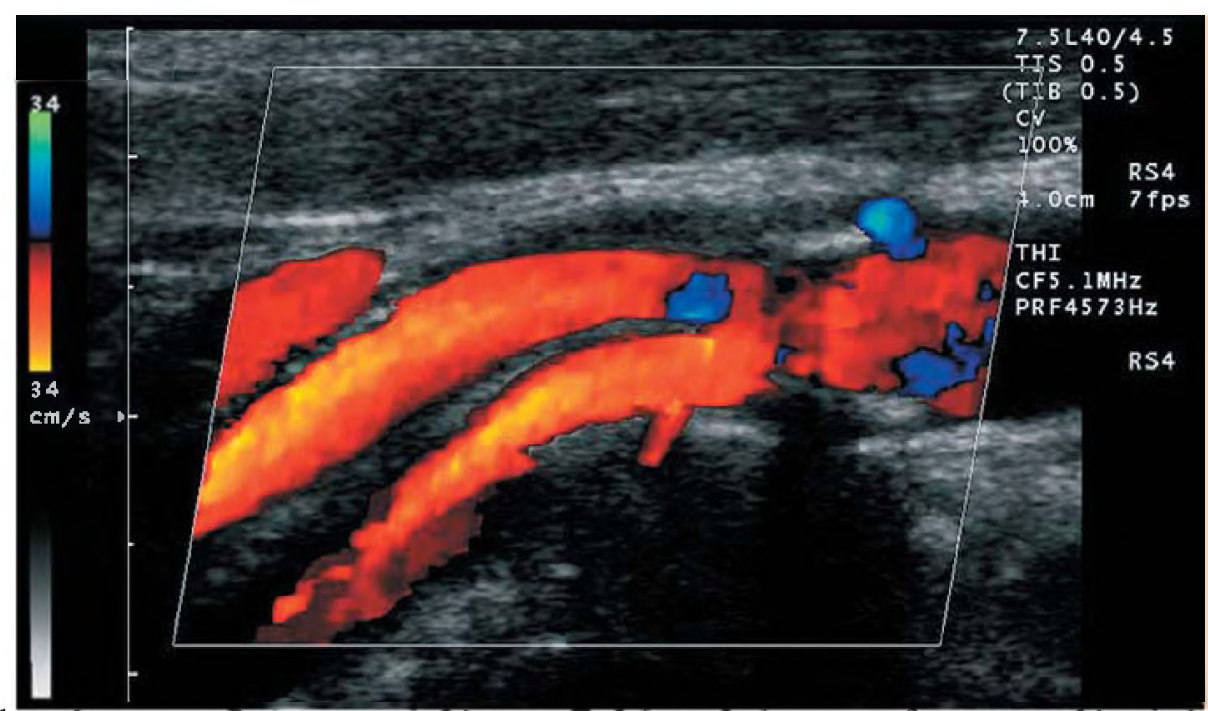

Figure 2. Color flow

Doppler image showing

blood flow in an artery.

The blue regions show regurgitation-blood flow reversed from the principal flow indicated in orange. (lmage from Siemens/Acuson, 2003.)

The echoes were first represented in so-called A-mode (amplitude) displays as vertical deflections on the screen of an oscilloscope whose beam scanned horizontally at a constant speed; the result was a series of blips.

Because tissue absorbs acoustic energy as heat, the later the blips occur, the smaller their amplitude. Assuming the speed of sound in human soft tissue has the constant value of $1540 \mathrm{~m} / \mathrm{s}$, one can interpret the time between reflections as the distance between tissue layers, and the relative amplitude changes can convey information about the impedance mismatch between one layer and the next. Some pathologies previously diagnosable only with surgery were identified using that early, noninvasive ultrasound technology.

The 1960s saw the development of commercial ultrasound systems that exploited the mass production of cathode-ray tubes. If A-mode amplitudes are represented by the brightness of dots on a phosphor screen and the transducer that generates the ultrasound pulses scans across the tissue, a B-mode (brightness) image results. Assuming, among other things, that the speed of sound is constant allows quantitative imaging of deep tissues, as seen in figure 1. The Quick Study on page 84 of March 2007 issue of Physics Today [Ref. 1] discusses the various assumptions that go into ultrasound imaging and the artifacts that arise if those assumptions are violated.

Modern ultrasound scanners use the linear superposition of spherical or cylindrical wavefronts of tiny piezoelectric crystals, usually lead zirconate titanate. Those crystals are arrayed to produce waveforms that can be steered or focused based upon the timing of the crystals' excitations. Both the emitted and received waves may be formed by suitably manipulating timing delays and adjustable gains in the individual elements' amplifiers. Thus a great deal of engineering goes into obtaining the image of a beating heart in an echocardiogram or of a fetus in a sonogram that may be parents' first glimpse of their new child.

\section{DOPPLER ULTRASOUND}

The Doppler effect is familiar to all who have heard the horn of an approaching train or considered the redshift of the expanding universe. In 1957 Shigeo Satomura demonstrated that the idea could be applied to ultrasound. If a sinusoidal acoustic frequency $\mathrm{f}$ is emitted by a fixed transducer located at an angle $\theta$ with respect to a blood vessel inside of which blood cells move with average velocity $\mathrm{V}$, the signals reflected to the transducer will be shifted in frequency by $\Delta \mathbf{f}=$ $(2 \mathrm{Vf} / \mathrm{c}) \cos \theta$, where $\mathrm{c}$ is the speed of sound in tissue. Thus for blood cells, the Doppler shift, measured by a continuouswave ultrasound probe transducer, can provide clinical information about blood flow.
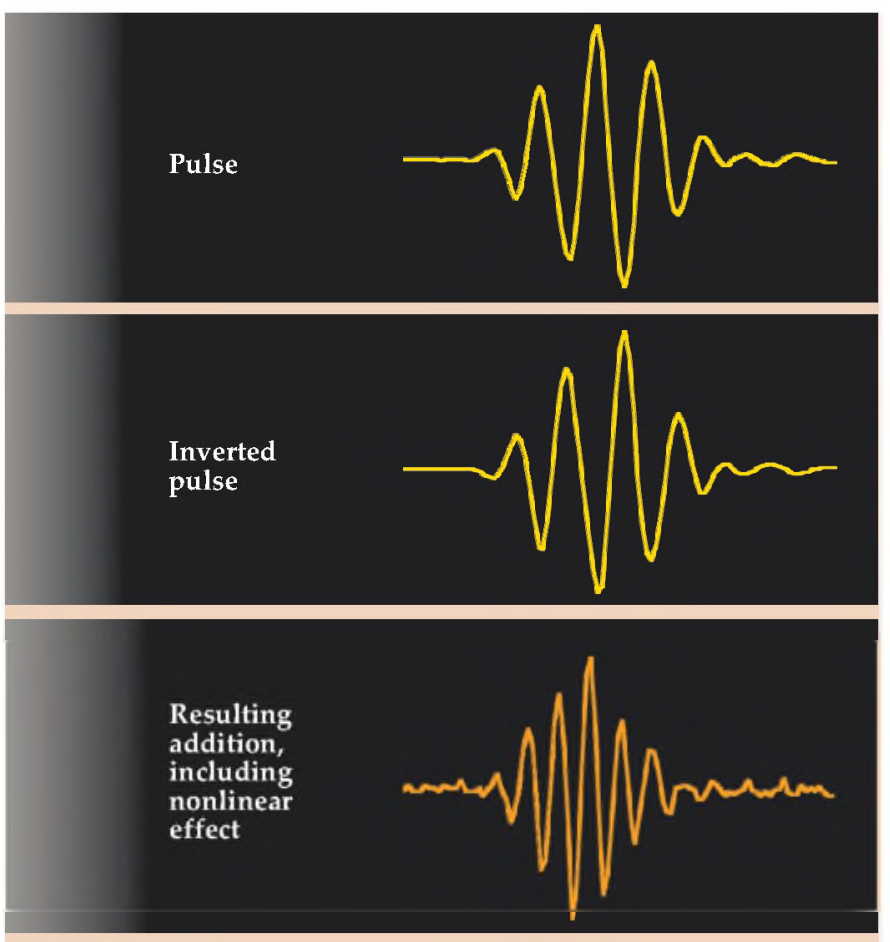

Figure 3. Tissue harmonic imaging relies on the nonlinear properties of tissue. A pulse and its inverse meet in tissue but because of nonlinearities the two pulses do not cancel. Instead, second-harmonic and other components arise. (Courtesy of Kirk D. Wallace, Washington University, St. Louis, Missouri.) 

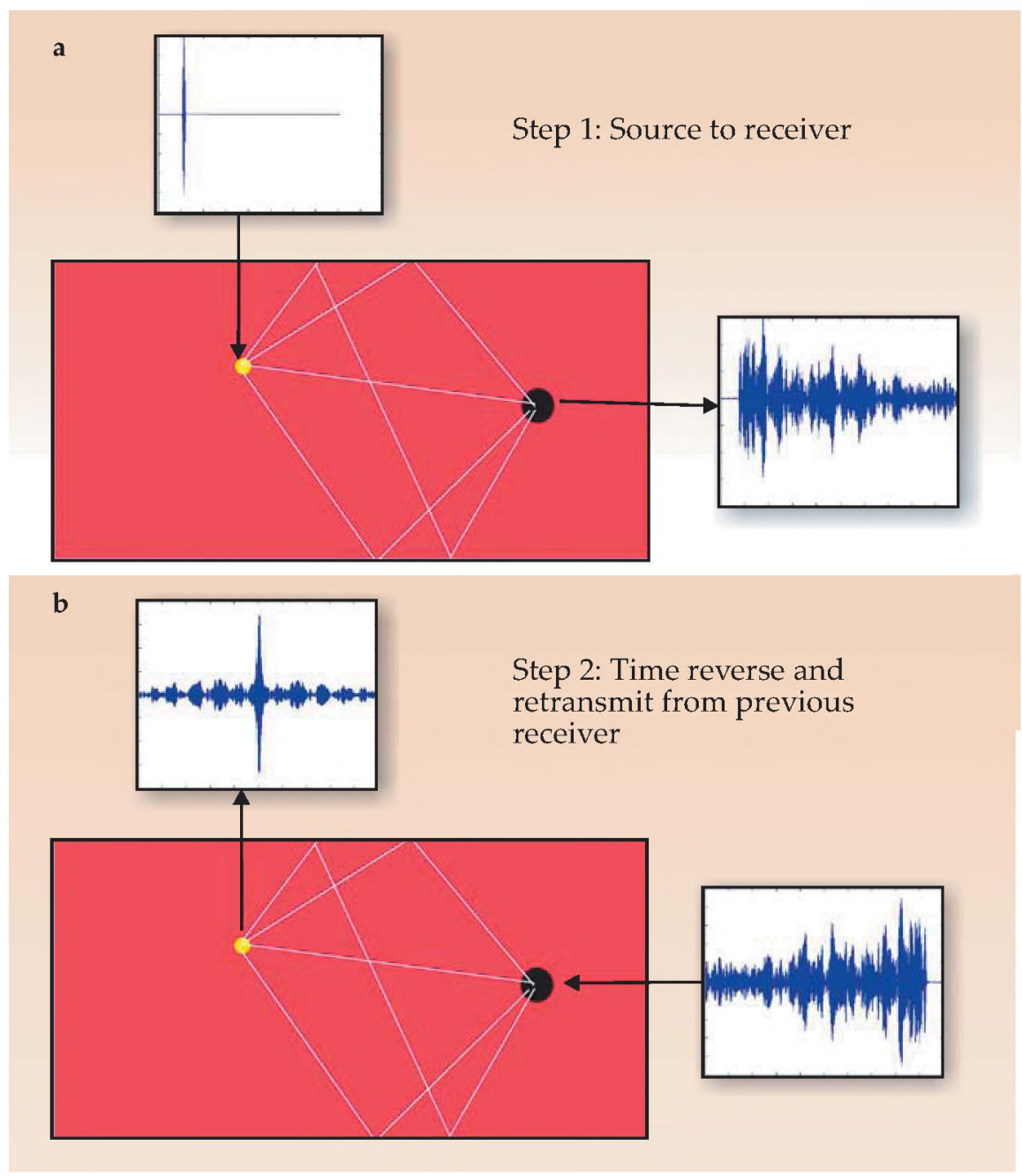

One complication, however, is that blood is effectively a non-Newtonian fluid whose viscosity is a strong function of shear flow rate. Its sound speed varies with red blood cell concentration, which differs between capillaries and larger vessels. Because red blood cells have a diameter of only about 7 microns - much smaller than the typical acoustic wavelengths of 750 to $150 \mu \mathrm{m}$ for $2-$ to $10-\mathrm{MHz}$ ultrasound - they cannot be resolved individually. Instead, the 250000 to 450000 red blood cells in each microliter act as an ensemble and scatter back to the transducer a fluctuating pattern of constructive and destructive interference called ultrasonic speckle. Nevertheless, for a continuous-wave Doppler system, the bulk motion of the flowing red blood cells typically provides a frequency shift that falls conveniently in the audio range. The characteristic squee-choo-squee-choo sound of the Doppler audio signal from the pulsatile flow of heartbeats is common in many hospitals and obstetricians' offices.

The biggest limitation of continuous-wave Doppler, however, is its spatial ambiguity. The difficulty arises because the Doppler signal is sensitive to all the vessels that intersect the beam. Pulsed-wave Doppler was developed in the 1970 s to provide high spatial resolution; dolphins, bats, and other animals use an analogous technique to locate and identify prey. Finite-length tone bursts are emitted whose
Figure 4. Time-reversal acoustics allows for impressive focusing of ultrasound pulses. (a) The yellow dot represents the region in which, ultimately, ultrasound is to be focused. But in the first step of TRA, that region acts as the source of a short burst. The signal received at the black dot is quite complicated. (b) Now the source is at the black dot and the initial waveform is the time reversal of the complicated signal received in panel $a$. The result is high temporal and spatial focusing at the yellow dot, the original source location. (Courtesy of Armen Sarvazyan, Artann Laboratories, West Trenton, New Jersey.)

durations correspond to the size of the volume to be interrogated. Unlike the continuous-wave case, for which the Doppler-shifted received frequency is compared with the transmitted one, in pulsed-wave Doppler each received echo is compared with a similar echo from the previous transmission. Because the pulse-repetition frequency is high, only small changes in tissue geometry and absorption occur from pulse to pulse. The result is relatively high sensitivity to overall arrangement of overlaying tissues.

In the 1980 s a signal-processing breakthrough allowed for the development of color flow Doppler, also called color flow imaging (CFI). At the time, clinicians knew that variations in blood flow velocities in an interrogated volume give rise not to a single Doppler shift, but rather to a whole spectrum of shifted frequencies. Fast-Fourier-transform methods for examining the spectra were too slow and the results too variable to be clinically useful. The new CFI technique estimated the average Doppler-shifted frequency by means of the small relative phase shifts from on reflected pulse to the next. Admittedly, those phase shifts result from slightly different sets of blood cells being present in the interrogated volume from one pulse to the next an so are not, strictly speaking, Doppler shifts of individual scatterers. Nevertheless, as long as the change in scatterer distribution varies only slightly, from pulse to pulse, CFI determines not only the average blood velocity but also a spectrum of velocities that can be color coded to represent forward and reverse blood flow. Such flow data are typically overlaid on the B-mode image in modern ultrasound scanners, as shown in figure 2.

One CFI variation that has found significant use in the past 10 years is so-called power Doppler, in which the integral over all frequencies of the square of the flow speed is shown as a color overlay on the B-mode image. Although information about the direction of flow is lost, the signal-to- 

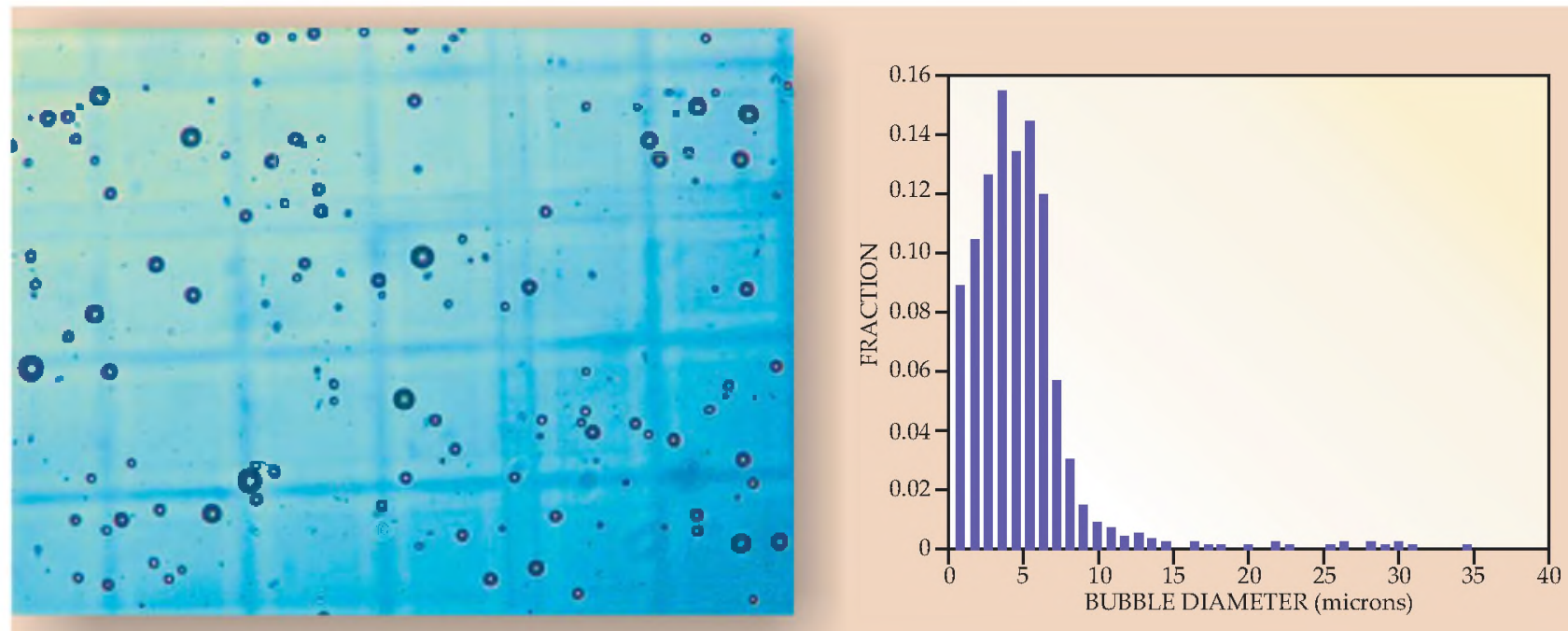

Figure 5. Microscopic bubbles are echo-contrast agents, strong reflectors of ultrasound. They may be used for diagnosis or may incorporate drugs that are delivered to specific sites. The microscope image to the left has a field of view roughly 300 microns across. The histogram to the right shows the size distribution of the bubbles seen in the micrograph. (Courtesy of Pierre D. Mourad and Shahram Vaezy, University of Washington, Seattle.)

noise ratio is increased so that power Doppler can detect tiny speed, or speed of tiny amounts of fluid. It can even image flow in capillaries.

Color flow imaging methods have contributed greatly to the success of ultrasound as a medically important modality. By providing a dynamic picture of blood flow, CFI Doppler has allowed physicians to diagnose leaking heart valves, vessel-blocking plaques and clots, and other abnormalities that would otherwise be difficult to detect.

\section{NONLINEAR ACOUSTICS}

The stiffness of biological tissue does not obey Hooke's law. As a consequence, the speed of sound varies locally with pressure and temperature and produces waveform distortion that compounds with propagation distance. If two wave-forms with different frequencies overlap in space and time, the tissue's essential nonlinearity produces sum and difference frequencies in the overlap region.

The acoustic nonlinearity parameter, defined as the ratio of the quadratic to the linear term in a Taylor-series expansion of pressure as a function of density, measures the deviation of the stiffness from Hooke's Law. Because the ratio is sensitive to subtle changes in material composition not reflected in acoustic impedance mismatches, a method of imaging the nonlinearity parameter could add complementary clinical information. To accomplish so-called nonlinear imaging of tissue, one can measure the strength of the sum or difference frequency by scanning a point where two ultrasound pulses intersect. For example, the sum or difference signal can be filtered and detected by a hydrophone or skin mounted sensor. Then the location of the coincident spot can be moved. The amplitude of the signal is proportional to the tissue nonlinearity at each point.

One relatively simple application of nonlinearity is tissue harmonic imaging. Developed in the late 1990s, the technique involves the combination of waveforms with the same frequency but opposite phase. Figure 3 shows a typical imaging pulse emitted from one piezoelectric element of an ultrasound transducer, with time on the horizontal axis and voltage, or pressure, on the vertical. If another array element is excited simultaneously with the inverse signal, the two pulses will mostly cancel each other's fundamental frequency where they overlap. Tissues nonlinearities, however, tend to move energy from the fundamental up to the second harmonic, which has twice the fundamental frequency. The effect of such distortion is slightly different for the pulse and its inverse, and the result is a reinforcement of the second harmonic. The focal area of the transducer, in sum, sees a pulse with a frequency and hence spatial resolution twice that of its B-mode equivalent and reflects that pulse back to the receiving elements of the transducer. Indeed, most ultrasound scanners in use today transmit signals at one frequency and listen to the returning echoes at twice that frequency, thus achieving better spatial resolution and improved signal-tonoise ratio.

To use nonlinear superposition, large-amplitude pulses must be made to intersect within the tissue volume. Since the late 1990 s, a techniques known as time-reversal acoustics (TRA) has provided a new tool to help accomplish that task. The basic idea is shown schematically in Figure 4. In the first step, an acoustic source produces a pulse that propagates along many different rays to a receiver; the result is a complicated impulse response. In the second step, the pattern of pulse arrivals at the receiver is reversed, and the receiver now acts as a transmitter. Because the wave equation is time-reversal invariant, the pulses follow the step-1 rays in reverse and end up coinciding at the original source as a highly spatially and temporally focused pulse. In effect, the tine-reversed signal at the receiver acts as an optimum 


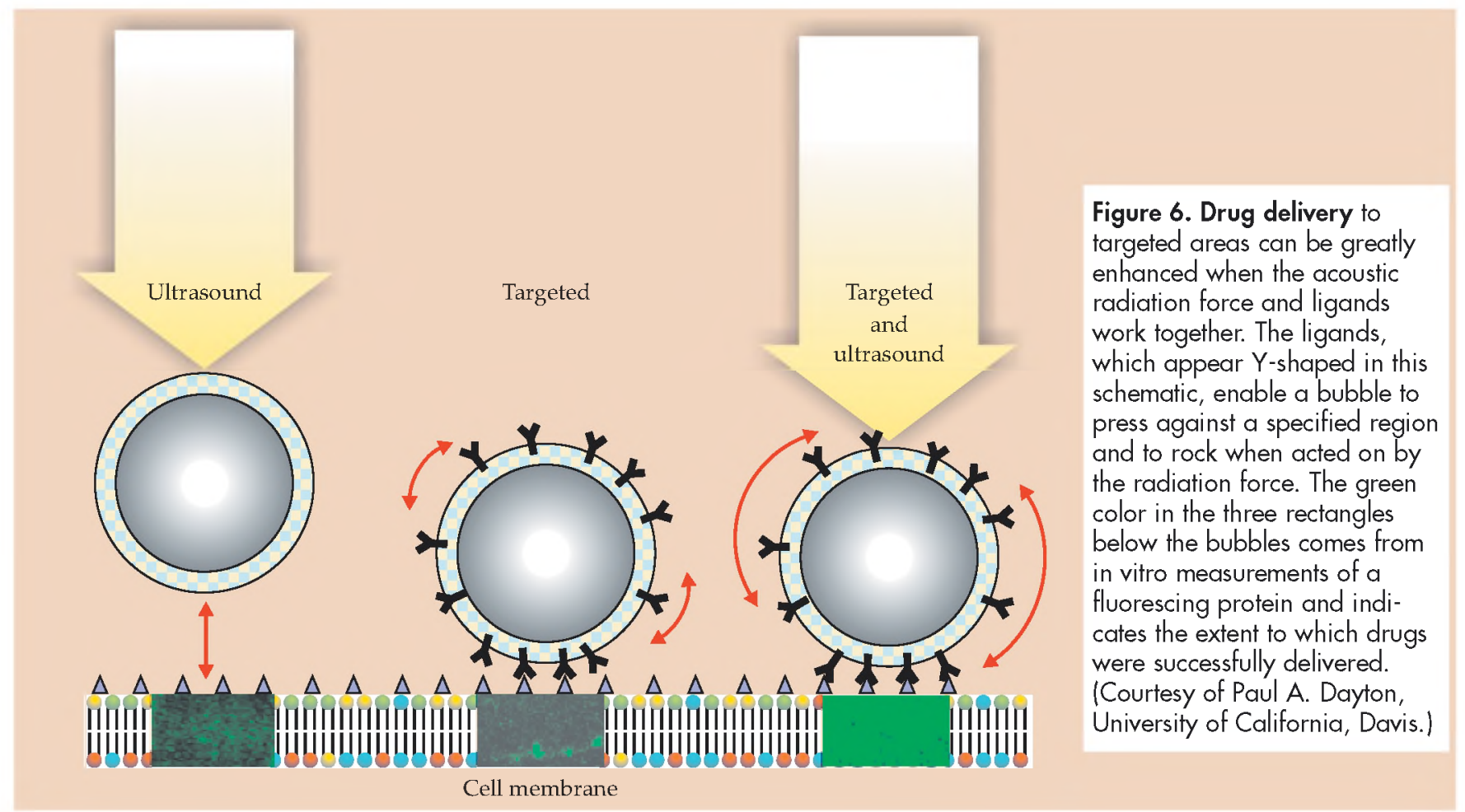

filter to deliver subwavelength focusing at the original source location.

In practice, TRA required that a source be placed in the body, although that source could be the tip of a biopsy need used to reflect incident ultrasound. If the receiver is not a single transducer but many transducers mounted on a surface, a highly focused spot can be generated at the site of the original source. TRA may be combined with other modalities to further improve focusing. One therapeutic application is to brain tumors at which ultrasound is applied. In that case, computed technology (CT) x-ray data can be used to compensate for the attenuation of the skull and the aberrations it causes to the acoustic field. Such dual-modality techniques are becoming more popular because techniques such as CT or magnetic resonance imaging provide information complementary to the mechanical parameters determined by ultrasound and thereby aid detection of pathology.

\section{TINY BUBBLES}

An increasingly important technique in diagnostic ultrasound involves blood-cell-sized microbubbles of perfluorocarbon gas, coated with proteins, lipids, or polymers (see figure 5). Such echo-contrast agents act as reflectors of ultrasound and are especially useful for quantifying blood flow in capillary beds or, when the bubbles' coatings contain biochemical markers called ligands, for helping doctors identify pathologies. Indeed, clot-sticking coatings may help not only to identify stroke-causing blood clots but also to treat them, since highamplitude ultrasound pulses may destroy bubbles and release clot-dissolving drugs.

Due to the acoustic impedance mismatch between bubbles and blood, bubbled reflect ultrasound strongly. The resulting reaction force, called the acoustic radiation force, pushes bubbles away from a transducer producing an ultrasound beam, or toward the pressure antinodes in an acoustic standing wave.

Each bubble has an acoustic resonance frequency related to the mass of the liquid around the bubble and the stiffness of the gas inside it. For an ultrasonic beam with a dominant frequency component near a bubble's resonance frequency, the acoustic radiation force can be strong enough to push the bubble to a vessel wall where drugs in the bubble coating can be released. As shown in figure 6, the combination of ligands to help the bubbles stick to the surface with an acoustic radiation force to press and wiggle the bubbles on the vessel wall enables a dramatic improvement in drug delivery compared with cases in which either the ultrasound or ligands are absent. A specific example of targeted drug delivery using ultrasound and bubbles is the delivery of chemotherapeutic agents to a tumor only, rather than systemically.

When a bubble is driven near resonance at a sufficiently large acoustic amplitude, its diameter can change appreciably during an acoustic cycle. If the maximum diameter is less than twice the undisturbed diameter, the bubble is said to undergo stable acoustic cavitation; its motion is mainly governed by the stiffness of the gas inside it. If the diameter more than doubles, the motion is controlled by the mass of the liquid surrounding the bubble; it undergoes inertial cavitation. Inertial cavitation bubbles commonly implode with such force that pressures thousands of atmospheres and temperatures of tens of thousands of Kelvin occur at their centers (see the article by Detlef Lohse in PHYSICS TODAY,

Contimued on Page 9 


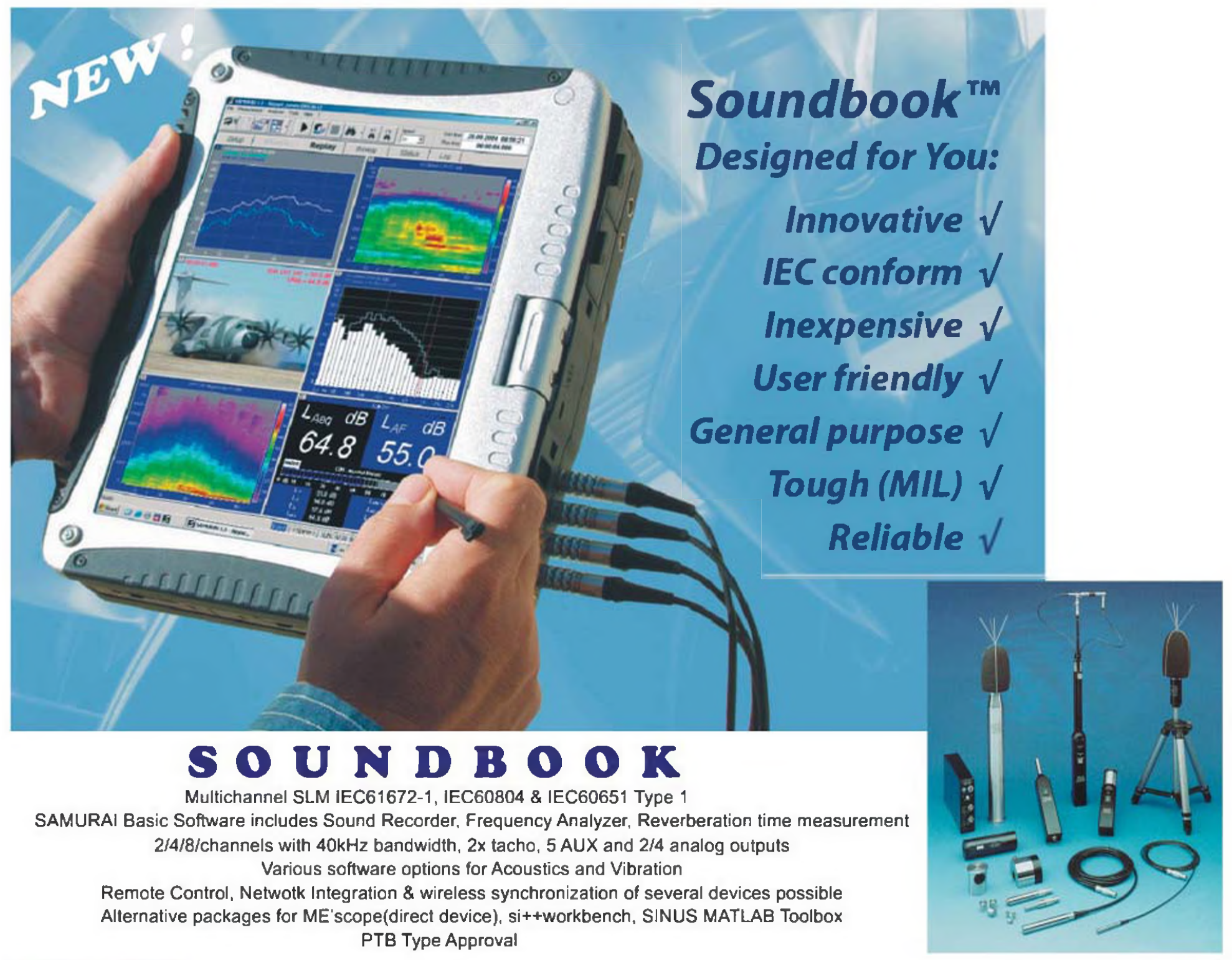

\section{SINUS}

G.R.A.S.

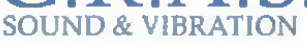

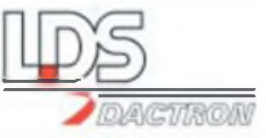

(3) O1dB-Stell

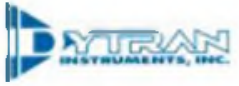

Metrolaser. Inc. th

I Vihrant Technology, Inc.

\section{Ottawa}

\section{3-598-0026}

info@noveldynamics.com

\section{Integrated Solutions from World Leaders}

Precision Measurement Microphones

Intensity Probes

Outdoor Microphones

Hydrophones

Ear Simulation Devices

Speech Simulation Devices

Calibrators

Array Microphones

Sound Quality

Sound Intensity

Sound Power

Room Acoustics

Noise Monitoring

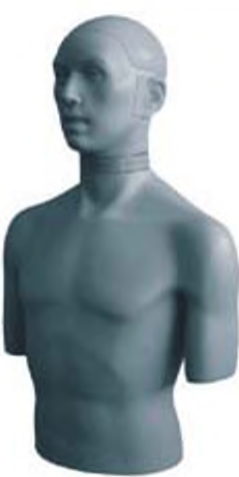

New KEMAR Manikin
Dynamic Signal Analyzers

Multi Channel Dynamic Analyzer/Recorders

Electro Dynamic Shaker Systems

Advanced Sound \& Vibration Level Meters

Doppler Laser Optical Transducers (Laser Vibrometers)

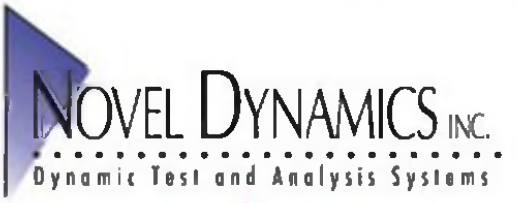

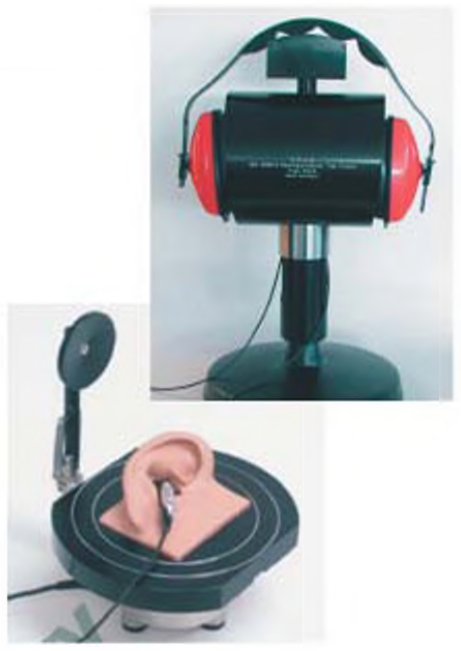

\section{Toronto}

519-853-4495

Ametelka@cogeco.ca 


\section{Continued from Page 7}

February 2003, page 36). Nearby tissues can be destroyed - a result to be avoided in diagnostic applications but one that can be useful for therapy. Nowadays, microbubble-ultrasound interactions also see applications that range from breaking kidney stones with acoustic shock waves (lithotripsy) to noninvasive removal of cholesterol plaque from arteries. If the echo-contrast bubbles span an appropriate size range, both kinds of cavitation may be present simultaneously. Because stable cavitation generates acoustic emissions below the beam frequency and inertial cavitation produces broadbandwidth acoustic noise at the moment of implosion, bubble activity can be detected and quantified. Such monitoring may make it possible to harness the potentially destructive effects of ultrasound for new therapies while avoiding adverse bioeffects in diagnostic applications, a greater understanding of the nonlinear mechanical properties of tissues, of bubbles, and of their interactions hold the promise of more advances in the medical use of ultrasound.

[Note: This article expands on a tutorial lecture first presen- ted on 17 October 2005 at the Acoustical Society of America meeting in Minneapolis, Minnesota]

\section{REFERENCES}

1. Kremkau, F. W. "Seeing is believing? Sonographic artifacts," Physics Today, 60(3), pp 84-85, 2007.

\section{ADDITIONAL READING}

F.V. Hunt, Origins in Acoustics: The Science of Sound from Antiquity to the Age of Newton, Yale U. Press, New Haven, CT (1978).

R.T. Beyer, Sounds of Our Times: Two Hundred Years of Acoustics, AIP Press/Springer, New York (1999).

T.L. Szabo, Diagnostic Ultrasound Imaging: Inside Out, Elsevier Academic Press, Burlington, MA (2004).

\begin{tabular}{|c|c|}
\hline $\begin{array}{l}\text { INSUL is an easy to } \\
\text { use software tool for } \\
\text { predicting airborne } \\
\text { sound insulation of } \\
\text { simple or complex } \\
\text { partitions consisting } \\
\text { of various materials \& }\end{array}$ & $\begin{array}{l}\text { SoundPLAN is a graphics } \\
\text { oriented noise prediction } \\
\text { program used for noise } \\
\text { planning, noise assessment } \\
\text { \& the development of noise } \\
\text { mitigation measures. The } \\
\text { database and management } \\
\text { structure allows for a quick }\end{array}$ \\
\hline
\end{tabular}



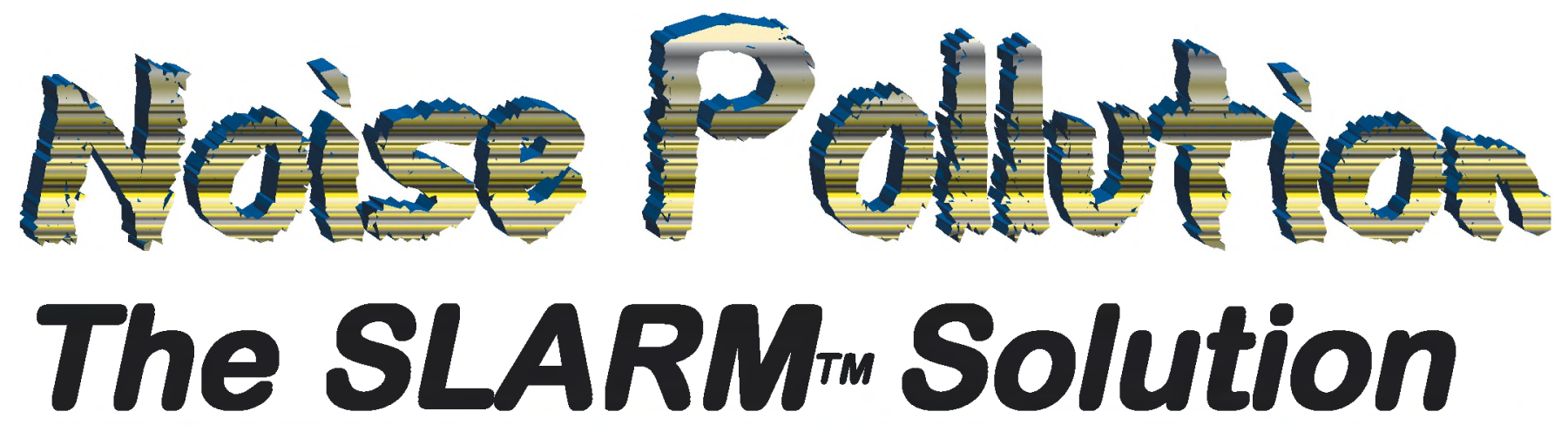

\section{PDA \& Laptop Displays Wired Wireless}
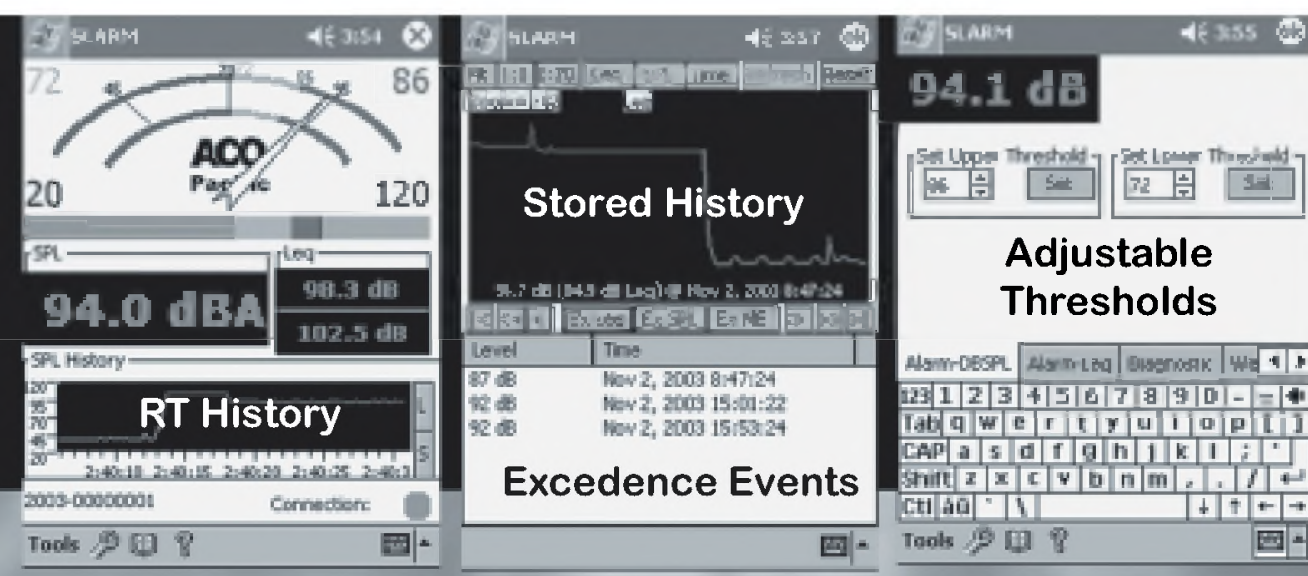

Adjustable

Thresholds
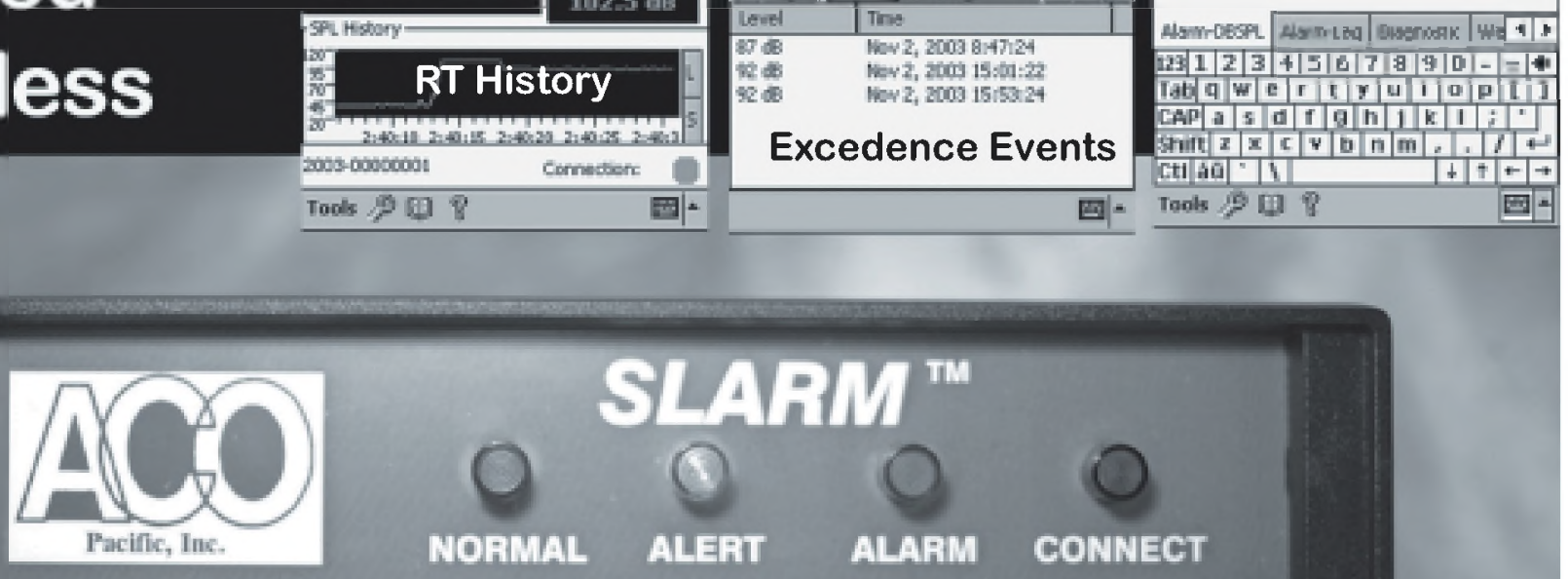

CONNECT

The SLARM ${ }^{\mathrm{TM}}$ developed in response to increased emphasis on hearing conservation and comfort in the community and workplace incorporates ACOustAlert ${ }^{\mathrm{TM}}$ and ACOustAlarm ${ }^{\mathrm{TM}}$ technology. Making the SLARM ${ }^{\mathrm{TM}}$ a powerful and versatile sound monitoring/alarm system.

Typical Applications Include: Community - Amphitheaters - Outdoor Events - Nightclubs/Discos - Churches -Classrooms

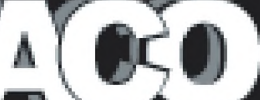
Pacific
Industrial - Machine/Plant Noise - Fault Detection - Marshalling Yards - Construction Sites - Product Testing WWW.acopacific.com
FEATURES

$\checkmark$ Wired and Wireless (opt)

$\sqrt{ }$ USB, Serial, and LAN(opt) Connectivity

$\checkmark$ Remote Display s and Programming

$\sqrt{ }$ SPL, Leq, Thresholds, Alert and Alarm

$\sqrt{ }$ Filters (A,C,Z), Thresholds, Calibration

$\sqrt{ }$ Multiple Profiles (opt)

$\sqrt{ } 100$ dB Display Range:

$\sqrt{ }$ 20-120 dBSPL and 40-140 dBSPL

$\sqrt{ }$ Real-time Clock/Calendar

$\sqrt{ }$ Internal Storage: 10+days @1/sec

$\sqrt{ }$ Remote Storage of $1 / 8$ second events

$\sqrt{ }$ 7052S Type $1.5^{\mathrm{TM}}$ Titanium Measurement Mic 2604 Read Ave., Belmont, CA 94002 Tel: 650-595-8588 FAX: 650-591-2891 acopac@acopacific.com ACOustics Begins With ACO ${ }^{\mathrm{TM}}$ 